Research Article

\title{
Comparative Genomics of the First and Complete Genome of "Actinobacillus porcitonsillarum" Supports the Novel Species Hypothesis
}

\author{
Valentina Donà and Vincent Perreten \\ Institute of Veterinary Bacteriology, Vetsuisse Faculty, University of Bern, Bern, Switzerland \\ Correspondence should be addressed to Vincent Perreten; vincent.perreten@vetsuisse.unibe.ch
}

Received 4 July 2018; Accepted 12 August 2018; Published 30 September 2018

Academic Editor: Marco Gerdol

Copyright (C) 2018 Valentina Donà and Vincent Perreten. This is an open access article distributed under the Creative Commons Attribution License, which permits unrestricted use, distribution, and reproduction in any medium, provided the original work is properly cited.

\begin{abstract}
"Actinobacillus porcitonsillarum" is considered a nonpathogenic member of the Pasteurellaceae family, which phenotypically resembles the pathogen Actinobacillus pleuropneumoniae. Previous studies suggested that "A. porcitonsillarum" may represent a new species closely related to Actinobacillus minor, yet no full genome has been sequenced so far. We implemented the Oxford Nanopore and Illumina sequencing technologies to obtain the highly accurate and complete genome sequence of the " $A$. porcitonsillarum" strain 9953L55. After validating our de novo assembly strategy by comparing the A. pleuropneumoniae S4074 ${ }^{\mathrm{T}}$ genome sequence obtained by Oxford Nanopore Technology combined with Illumina reads with a PacBio-sequenced S4074 ${ }^{\mathrm{T}}$ genome from the NCBI database, we performed comparative analyses of the 9953L55 genome with the A. minor type strain NM305 ${ }^{\mathrm{T}}$, A. minor strain 202, and A. pleuropneumoniae $\$ 4074^{\mathrm{T}}$. The 2,263,191 bp circular genome of $9953 \mathrm{~L} 55$ consisted of 2168 and 2033 predicted genes and proteins, respectively. The lipopolysaccharide cluster resembled the genetic organization of A. pleuropneumoniae serotypes 1,9 , and 11 , possibly explaining the positive reactions observed previously in serotyping tests. In contrast to $\mathrm{NM} 305^{\mathrm{T}}$, we confirmed the presence of a complete apxIICABD operon in 9953L55 and 202 accounting for their hemolytic phenotype and Christie-Atkins-Munch-Petersen (CAMP) reaction positivity. Orthologous gene cluster analysis provided insight into the differential ability of strains of the A. minor/"porcitonsillarum" complex and A. pleuropneumoniae to ferment lactose, raffinose, trehalose, and mannitol. The four strains showed distinct and shared transposable elements, CRISPR/ Cas systems, and integrated prophages. Genome comparisons based on average nucleotide identity and in silico DNA-DNA hybridization confirmed the close relationship among strains belonging to the A. minor/"porcitonsillarum" complex compared to other Actinobacillus spp., but also suggested that 9953L55 and 202 belong to the same novel species closely related to A. minor, namely, "A. porcitonsillarum." Recognition of the taxon as a separate species would improve diagnostics and control strategies of pig pleuropneumonia.
\end{abstract}

\section{Introduction}

"Actinobacillus porcitonsillarum" is a Gram-negative rod belonging to the Pasteurellaceae family, which is regularly isolated from the tonsils of healthy pigs and phenotypically resembles Actinobacillus pleuropneumoniae, the causative agent of porcine pleuropneumonia, which is associated with high economic burdens in the pig industry worldwide $[1,2]$. "A. porcitonsillarum" mimics the major antigenic factors of A. pleuropneumoniae causing cross-reactivity in serological tests [1], which negatively affects serological diagnosis of A. pleuropneumoniae, potentially leading to the unnecessary depopulation and/or antimicrobial treatment of pig herds.

The "A. porcitonsillarum" strain 9953L55 (CCUG 46996) was firstly isolated from the tonsils of a healthy pig belonging to a high-health status herd considered to be free from $A$. pleuropneumoniae, in which regular serological testing suddenly evidenced a low number of pigs showing weak positive reactions for A. pleuropneumoniae serogroups 1, 9, and 11 [3]. Subsequent phenotypic and biochemical analyses indicated that this strain appeared to be identical to $A$. 
pleuropneumoniae, including the hemolytic growth on blood agar plates and the Christie-Atkins-Munch-Petersen (CAMP) activity, i.e., a cohemolytic effect observed on blood agar plates in the presence of a sphingomyelinase ( $\beta$-hemolysin)-producing Staphylococcus aureus, but with the exception that it did not ferment mannitol [1]. Serotyping by three different methods showed a positive reaction with antiserum raised against serotype $1 \mathrm{~A}$. pleuropneumoniae S4074 ${ }^{\mathrm{T}}$ [1]. Nevertheless, three A. pleuropneumoniae-specific PCRs were negative, indicating also the absence of the $a p x I V$ gene, which was previously proven to be species-specific for A. pleuropneumoniae [1,4]. Toxin gene typing PCR for the major RTX toxins (ApxI, ApxII, and ApxIII) additionally revealed that $a p x I I$, but not $a p x I$ or $a p x I I I$ genes were present [1].

Phylogenetic analysis of the 16S rRNA gene sequence indicated that "A. porcitonsillarum" was most closely related to Actinobacillus minor strain 202 (formerly named "Haemophilus strain 202", but subsequently classified as a borderline A. minor strain [5]), and to the A. minor type strain $\mathrm{NM} 305^{\mathrm{T}}$, although it distinguished itself phenotypically from the latter by the hemolysis and CAMP activity [1]. Interestingly, a later study provided evidence that $A$. minor 202 also produced the ApxII toxin and appeared to be genetically rather more related to "A. porcitonsillarum" than to A. minor NM305 ${ }^{\mathrm{T}}[6]$.

Despite these previous observations suggesting that " $A$. porcitonsillarum" may represent a new species, it has not been recognized as a distinct species so far, mainly due to the absence of sufficient phenotypic markers to distinguish it from A. minor [7]. However, a clear differentiation of the commensal " $A$. porcitonsillarum" from the pathogen $A$. pleuropneumoniae would be essential in diagnostics and, particularly, in eradication programs.

To corroborate these previous observations at a genomic level, we used the Oxford Nanopore and Illumina sequencing technologies to sequence the " $A$. porcitonsillarum" strain 9953L55, which was proposed as a type strain for " $A$. porcitonsillarum." After the validation of our de novo assembly approach by obtaining the genome of the A. pleuropneumoniae strain $\mathrm{S} 4074^{\mathrm{T}}$ with the Oxford Nanopore Technology combined with Illumina reads and comparing it with the PacBio-sequenced genome of the same strain found in the NCBI database, we implemented this method to obtain the highly accurate circular genome sequence of strain 9953L55, which was further used for comparative analyses with the genome sequences of $A$. minor 202, A. minor $\mathrm{NM} 305^{\mathrm{T}}$, and A. pleuropneumoniae $\mathrm{S} 4074^{\mathrm{T}}$.

\section{Materials and Methods}

2.1. Bacterial Strains, Growth Conditions, and Sugar Fermentation Test. "A. porcitonsillarum" 9953L55 (CCUG 46996), A. minor NM305 ${ }^{\mathrm{T}}$ (CCUG $38923^{\mathrm{T}}$ ), and A. pleuropneumoniae $\mathrm{S} 4074^{\mathrm{T}}$ (ATCC $27088^{\mathrm{T}}$ ) were grown on chocolate agar plates supplemented with Polyvitex (BioMérieux) at $37^{\circ} \mathrm{C}$ with $5 \% \mathrm{CO}_{2}$. Lactose, raffinose, and trehalose fermentation was assessed, using $\mathrm{S} 4074^{\mathrm{T}}$ and $\mathrm{NM} 305^{\mathrm{T}}$ as control strains, in PPLO broth (Difco) supplemented with $40 \mu \mathrm{g} / \mathrm{ml}$ NAD, as described previously $[1,8]$.

2.2. DNA Isolation and Sequencing. DNA was isolated with a modified phenol/chloroform extraction method, treated for $30 \mathrm{~min}$ with $0.5 \mu \mathrm{l}$ RNase $(20 \mathrm{mg} / \mathrm{ml})$ (Qiagen), and purified with 0.8X Agencourt AMPure beads (Beckman Coulter) [9]. The purified DNA was subsequently sheared to $8-10 \mathrm{~kb}$ fragments with a g-TUBE (Covaris), and library preparation was performed with the SQK-LSK108 1D ligation sequencing kit (Oxford Nanopore), as per the manufacturer's instructions. The sequencing library was sequenced on a R9.4 SpotON flow cell (Oxford Nanopore) with the MinION Mk $1 \mathrm{~B}$ sequencing device (Oxford Nanopore) for 24 hours. In parallel, the DNA was also submitted to GATC, Constance, Germany, for $2 \times 150$ paired-end sequencing on an Illumina HiSeq (Illumina) platform.

2.3. Genome Assembly. Base calling and quality filtering of the Oxford Nanopore Technology (ONT) reads were performed with Albacore v2.0.1. Pairing, trimming, and quality filtering of the Illumina reads were performed with Trimmomatic v0.33. ONT reads were assembled with Canu v1.3 with default parameters and the option corOutCoverage $=100$ [10]. Paired-end Illumina reads were mapped to the Canugenerated scaffold with BWA-MEM v0.7.13 and polished with Pilon 1.22 twice [11]. A third mapping of the Illumina reads was performed with BWA-MEM for the final inspection and curation of the polished sequence with the Geneious software v10.2.3 (Biomatters). In case of repetitive regions leading to unbalanced (low) read coverage, these regions were extracted to locally repeat read mapping with BWAMEM. The final circular genome sequences of strains 9953L55 and S4074 ${ }^{\mathrm{T}}$ were first annotated with Prokka v1.12 for primary sequence analysis and subsequently with the NCBI prokaryotic genome annotation pipeline [12]. Paired-end Illumina reads were used to run plasmidSPAdes v3.9.0 with default parameters [13].

2.4. Genome Analysis and Comparison. The whole-genome shotgun and complete genome sequences, which were retrieved from the NCBI database for the genome comparisons, are deposited under the following GenBank

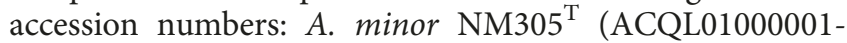
ACQL01000197), A. minor 202 (ACFT01000001ACFT01000154), A. pleuropneumoniae S4074 ${ }^{\mathrm{T}}$ (PacBio, CP029003; Roche 454, ADOD01000001-ADOD01000044), A. equuli $19392^{\mathrm{T}}$ (CP007715), A. succinogenes $130 \mathrm{Z}^{\mathrm{T}}$ (NC_009655), A. suis ATCC 33415 ${ }^{\mathrm{T}}$ (NZ_CP009159), A. ureae ATCC $25976^{\mathrm{T}}$ (AEVG01000001-AEVG01000183), A. capsulatus DSM $19761^{\mathrm{T}}$ (ARFN01000001-ARFN01000049), and $A$. seminis ATCC $15768^{\mathrm{T}}$ (NLFK01000001NLFK01000022). Genome alignments were performed with progressiveMauve v2.3.1 [14]. OrthoVenn was used to identify orthologous genes [15]. Online available platforms were used to characterize the presence of known resistance genes (ResFinder) [16], plasmids (PlasmidFinder) [17], insertion sequences (IS, ISfinder) [18], clustered regularly interspaced short palindromic repeat (CRISPR) arrays and CRISPR- 


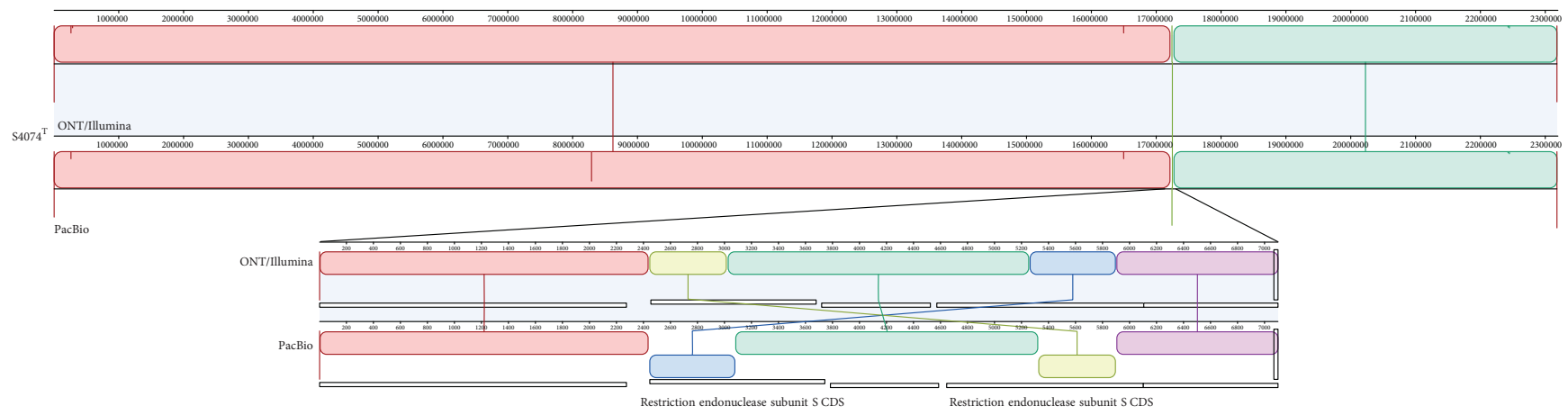

FIgURE 1: MAUVE alignment of the genome sequence of A. pleuropneumoniae S4074 ${ }^{\mathrm{T}}$ obtained by Oxford Nanopore Technology (ONT)/ Illumina (top) and PacBio (bottom) sequencing. The same color boxes, i.e., locally collinear blocks (LCB), represent homologous regions of sequence without rearrangement. The inset underneath magnifies the only rearrangement found between the two sequences.

associated gene (CRISPR/Cas) systems (CRISPRone) [19], and phage sequences (PHASTER) [20]. The circular map of 9953L55 including the BLAST-based comparison with the genome sequences of $A$. minor $\mathrm{NM} 305^{\mathrm{T}}, A$. minor 202, and A. pleuropneumoniae $\mathrm{S} 4074^{\mathrm{T}}$ was generated with the BLAST Ring Image Generator (BRIG) [21]. Comparisons of average nucleotide identity (ANI) based on BLAST and MUMmer pairwise sequence alignments (ANIb and ANIm, respectively) were obtained with JSpeciesWS [21,22]. The distance matrix representing the ANI divergence (defined as $100 \%$ - ANI) was used to compute a complete linkage hierarchical clustering with the hclust function in $\mathrm{R} v 3.0 .1$, as done previously [23]. In silico DNA-DNA hybridization (is DDH) based on genome BLAST distance phylogeny was performed with GGDC 2.1 [24]. Only results based on formula 2 were used for analysis, since it estimates is DDH values independently of genome length and is therefore recommended for incomplete genomes $[25,26]$.

2.5. Nucleotide Sequence GenBank Accession Number. The complete nucleotide sequences of the "A. porcitonsillarum" strain 9953L55 and of the A. pleuropneumoniae strain S $4074^{\mathrm{T}}$ were deposited in DDBJ/EMBL/GenBank under the accession numbers CP029206 and CP030753, respectively.

\section{Results and Discussion}

After base calling and quality filtering, 580,932 $1 \mathrm{D}$ pass ONT reads corresponding to $4.08 \mathrm{Gbp}(>1800 \mathrm{X}$ coverage) and $10,478,015$ paired-end Illumina reads were obtained for the A. pleuropneumoniae strain S4074 ${ }^{\mathrm{T}}$. Assembly of the ONT reads generated a single $2.32 \mathrm{Mbp}$ contig with overlapping ends, which was circularized and polished with paired-end Illumina reads.

Alignment of the obtained $54074^{\mathrm{T}}$ genome with the complete genome sequence of the same strain previously sequenced with PacBio technology, which we retrieved from the NCBI database (accession number CP029003), indicated a very high sequence homology (Figure 1). Only one rearrangement was identified between the two genome sequences, which mapped to the $5^{\prime}$-end region of two genes in opposite orientation both encoding a restriction endonuclease subunit $S$ (Figure 1). High sequence divergence was observed solely in a $5 \mathrm{kbp}$ region comprising 5 genes encoding a $\mathrm{D}$-alanine-D-alanine ligase, cell division proteins ( fts Q, ftsA, and ftsZ), and the UDP-3-O-[3-hydroxymyristoyl] $\mathrm{N}$-acetylglucosamine deacetylase $\left(l_{p} \times C\right)$. All 5 genes are annotated as frameshifted and contain internal stop codons in the PacBio-generated S4074 ${ }^{\mathrm{T}}$ genome sequence. In contrast, all genes were intact in the genome obtained by ONT/Illumina sequencing, and a comparison with a previous whole-genome shotgun assembly of the same strain obtained by Roche 454 sequencing technology, which we retrieved from the NCBI database (accession number ADOD01000001-ADOD01000044), showed 100\% identity. A $5 \mathrm{bp}$ indel was found in a gene encoding a methyltransferase annotated as incomplete in the PacBio-generated genome sequence, restoring the completeness of the gene in the genome obtained by ONT/Illumina sequencing. Only 2 additional single nucleotide polymorphisms (SNPs) and one indel (in an intergenic region) were identified between the two genomes.

Taken together, these results confirmed that our approach, i.e., de novo assembly of ONT reads combined with Illumina polishing, can be successfully applied to generate a complete and highly accurate bacterial genome sequence. Therefore, this strategy was further used to obtain the full genome sequence of the "A. porcitonsillarum" strain 9953L55.

After base calling and quality filtering, 721,267 1D pass ONT reads corresponding to $4.82 \mathrm{Gbp}$ ( $>2000 \mathrm{X}$ coverage) and 5,367,150 paired-end Illumina reads were obtained for strain 9953L55. Assembly of the ONT reads generated a single $2.26 \mathrm{Mbp}$ contig with overlapping ends, which was circularized and polished with paired-end Illumina reads to obtain the complete circular sequence of the " $A$. porcitonsillarum" 9953L55 chromosome, as done for $\mathrm{S}_{4074}{ }^{\mathrm{T}}$. In silico analysis with PlasmidFinder and plasmidSPAdes using paired-end Illumina reads suggested the absence of plasmids.

The circular genome of 9953L55 consisted of $2,263,191 \mathrm{bp}$ with an average $39.7 \%$ GC content and displayed, as expected, a high nucleotide sequence similarity with the genome sequences of $A$. minor $\mathrm{NM}^{\mathrm{T}} 5^{\mathrm{T}}$ and 202, while A. pleuropneumoniae $\mathrm{S} 4074^{\mathrm{T}}$ was more dissimilar 

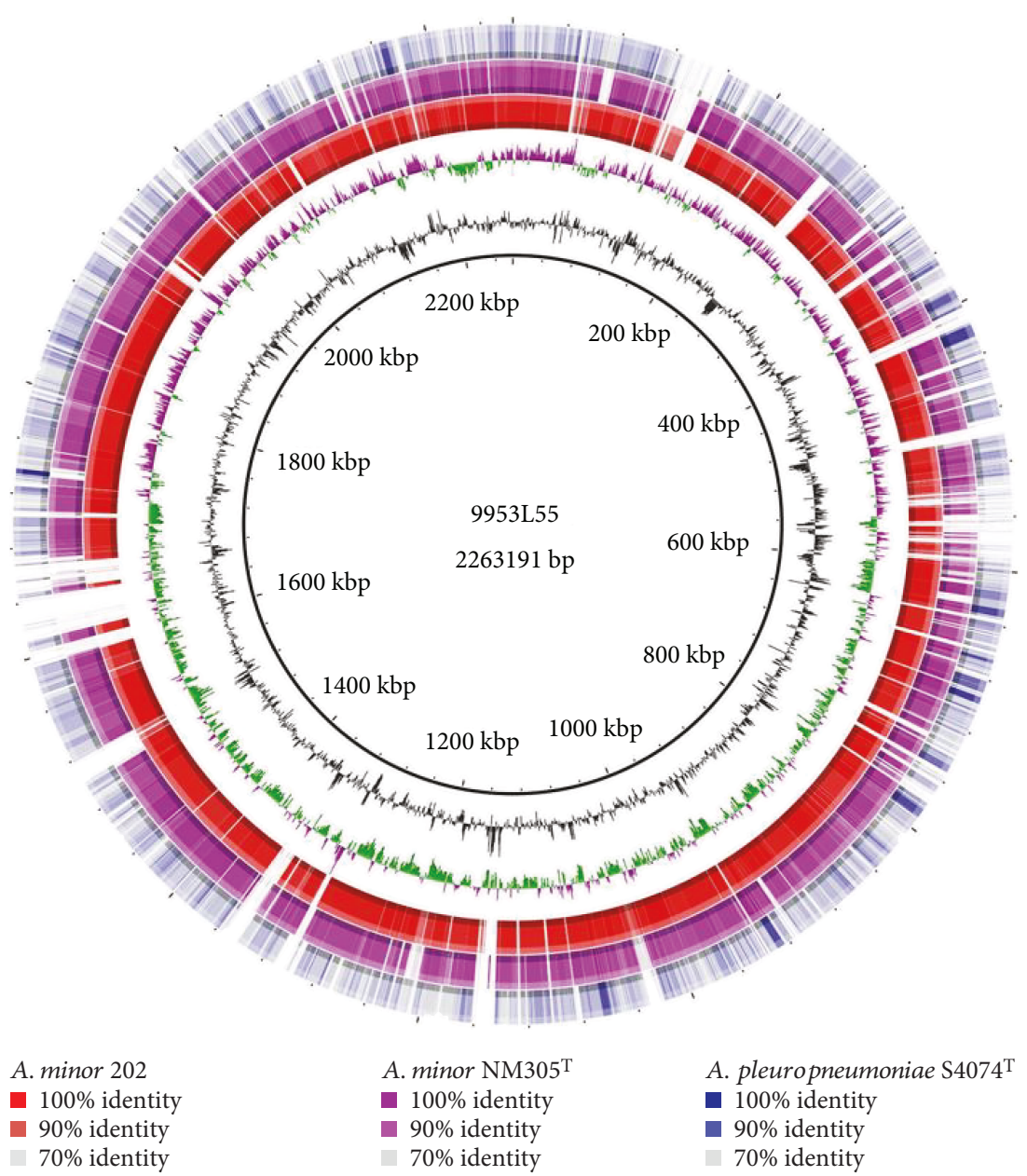

A. pleuropneumoniae $\mathrm{S} 4074^{\mathrm{T}}$
100\% identity
90\% identity
$70 \%$ identity

FIgURE 2: Circular map of the "A. porcitonsillarum" strain 9953L55. The scale ring shows the coordinates in kilobase pairs. The second ring represents the average GC content. The third ring represents the GC skew. The colored outer rings display regions of homology based on BLASTn. First outer ring (red): A. minor 202, second outer ring (pink): A. minor $\mathrm{NM}_{305^{\mathrm{T}}}$, and third outer ring (blue): $A$. pleuropneumoniae $\mathrm{S} 4074^{\mathrm{T}}$.

(Figure 2). In total, 2168 genes, including six copies of the rrn operon encoding the 16S, 23S, and 5S rRNA, as well as 2087 CDS, of which 2033 encode proteins and 54 are pseudogenes, were predicted.

Analysis with OrthoVenn showed that the four strains display 1523 common clusters of orthologous genes (COGs), of which 1507 were single-copy clusters, indicating few duplication events before speciation (Figure 3). The two $A$. minor strains and "A. porcitonsillarum" 9953L55 shared 130 additional COGs, reflecting their closer phylogenetic relationship compared to $A$. pleuropneumoniae, as suggested previously [1]. Interestingly, most of the COGs present only in $9953 \mathrm{~L} 55$ and $\mathrm{S} 4074^{\mathrm{T}}$ were genes belonging to the lipopolysaccharide (LPS) cluster, which was located (as in the other three strains) between the $\operatorname{erp} A$ and $r p s U$ genes and closely resembled the genetic organization of the A. pleuropneumoniae serotype 1, 9, and 11 LPS cluster. This may explain the previously observed cross-reactivity with antiserum against $\mathrm{S} 4074^{\mathrm{T}}$ and, particularly, the positive reaction in the dotELISA test with a monoclonal antibody recognizing a common O-chain LPS epitope of A. pleuropneumoniae serotypes 1,9 , and $11[1]$.
The further analysis of the COGs shed some light on the different phenotypes observed in the biochemical tests $[1,5,27]$.

RTX toxins (ApxI, ApxII, and ApxIII) in A. pleuropneumoniae are responsible for its hemolytic activity and CAMP positivity $[28,29]$. Orthologs for the apxIICA genes were identified in all strains but not in $A$. minor $\mathrm{NM} 305^{\mathrm{T}}$. In fact, an intact and a complete $a p x I I C A B D$ operon was located between the aspC and folC genes in both " $A$. porcitonsillarum" 9953L55 and A. minor 202, but no apxI, apxIII, or apxIVA genes were found, consistent with previous observations $[1,7,30]$. This apxIICABD operon was shown to be responsible and sufficient for RTX toxin ApxII expression and secretion and, consequently, for their hemolytic phenotype [7, 30].

Regarding the main differences in sugar utilization, orthologs encoding the $\beta$-galactosidase were present in all four strains accounting for their positive reaction with the o-nitrophenyl- $\beta$-D-galactopyranoside (ONPG) test $[1,5]$. However, a full lac operon, i.e., including genes encoding the transcriptional regulator (lacI), the lactose permease $(\operatorname{lac} Y)$, and the $\alpha$-galactosidase (melA), was identified only 

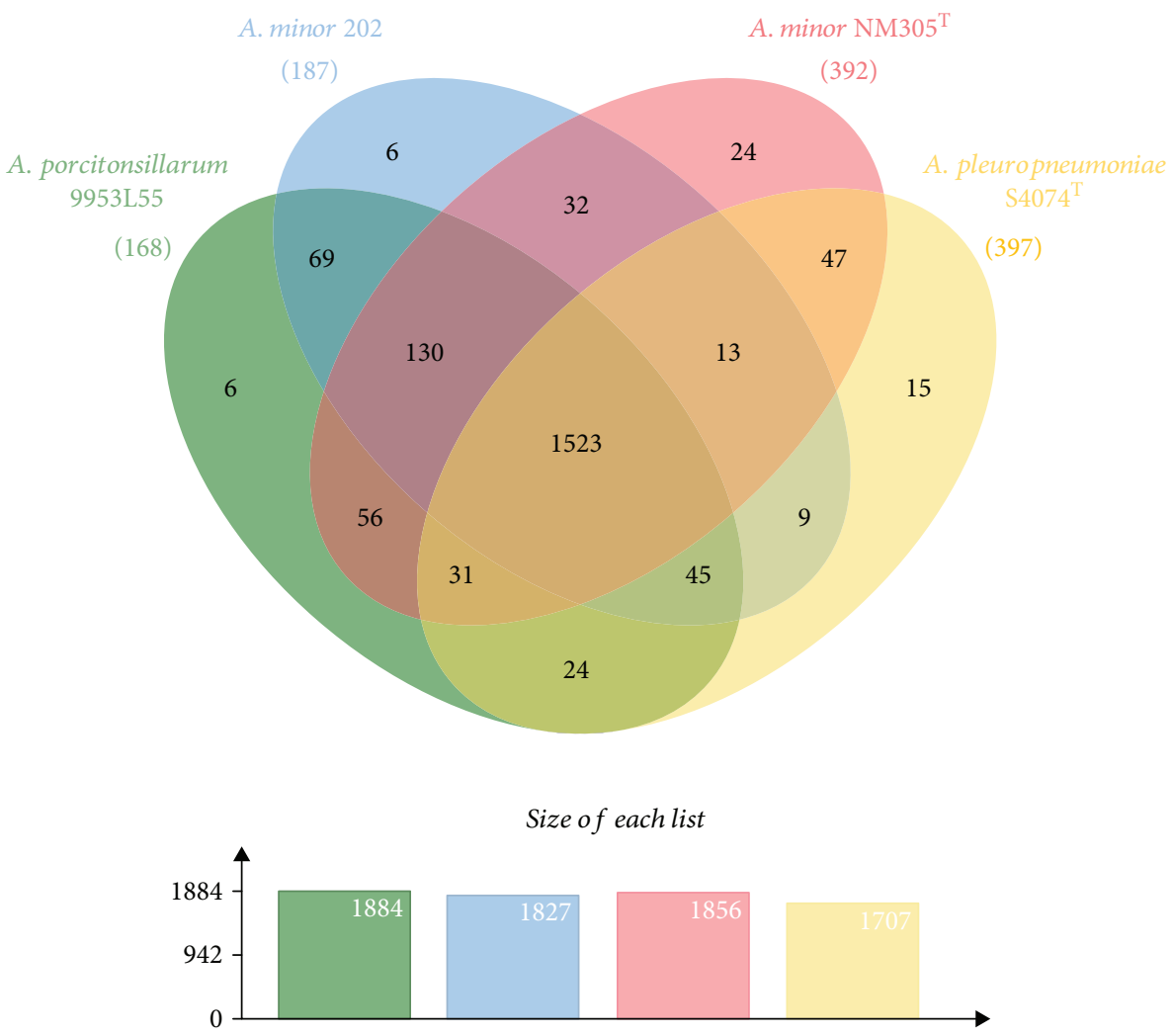

FIGURE 3: Venn diagram depicting clusters of orthologous genes (COGs) in “A. porcitonsillarum" 9953L55, A. minor 202, A. minor NM305" and A. pleuropneumoniae S4074 ${ }^{\mathrm{T}}$. The number of singletons for each strain is shown in brackets. The total number of COGs for each strain is displayed in the graph underneath.

in the "A. porcitonsillarum" and in both A. minor strains. The absence of the lactose permease and the $\alpha$-galactosidase provides an explanation for the inability of $A$. pleuropneumoniae to ferment lactose and raffinose, respectively, in contrast to most A. minor strains $[5,27]$. Consistently, we confirmed by testing lactose and raffinose fermentation that " $A$. porcitonsillarum" 9953L55 also produces acid from both sugars.

While A. pleuropneumoniae does not ferment trehalose, most A. minor strains are trehalose fermenters [5]. We identified a full tre operon with genes encoding the HTH transcriptional regulator (treR), the PTS trehalose transporter (treP), and the trehalose-6-phosphate hydrolase (treA) only in "A. porcitonsillarum" 9953L55 and A. minor $\mathrm{NM} 305^{\mathrm{T}}$, suggesting that both strains are able to import and ferment trehalose. As expected, when testing their ability to utilize trehalose, acid production was observed for both 9953L55 and NM305 ${ }^{\mathrm{T}}$, but not for S4074 ${ }^{\mathrm{T}}$.

On the other hand, we found no orthologs in " $A$. porcitonsillarum" $9953 \mathrm{~L} 55$ and in both A. minor strains for the $m t l D$ and $m t l A$ genes, which code for the PTS mannitol transporter and the mannitol-1-phosphate-5-dehydrogenase in $A$. pleuropneumoniae, respectively, providing an explanation for their inability to assimilate and/or ferment mannitol $[1,5]$.

Most COGs shared only by "A. porcitonsillarum" 9953L55 and A. minor 202 were genes involved in different metabolic pathways, iron transport, response to stimuli, and quorum sensing. However, we also identified many orthologs for genes related to the CRISPR/Cas system, which represents the bacterial adaptive immune system against phages. Further analysis showed that both 9953L55 and 202 possess a subtype I-C CRISPR/Cas system, including a CRISPR array containing 37 repeat units in the "A. porcitonsillarum" strain. In contrast, complete subtypes II-C and I-F (yet in a particular genetic rearrangement) were identified in $A$. minor $\mathrm{NM} 305^{\mathrm{T}}$ and $A$. pleuropneumoniae $\mathrm{S} 4074^{\mathrm{T}}$, respectively.

Regarding phages, only one intact HP2-related Haemophilus prophage and an incomplete prophage region of $6.2 \mathrm{~kb}$ were identified in "A. porcitonsillarum" 9953L55 between positions 1,610,930-1,645,906 and 1,650,4251,656,579, respectively. Interestingly, different intact Haemophilus as well as enterobacterial prophages were found

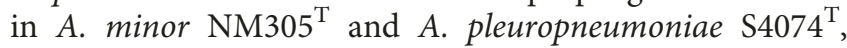
but not in A. minor 202 (data not shown).

Among the COGs identified exclusively in " $A$. porcitonsillarum" 9953L55 and A. minor NM305" the ISApl1 was the most abundant with 10 copies present in the genome of the "A. porcitonsillarum" strain. This IS is typically found in Actinobacillus spp. and has been recently associated with the widespread of the colistin-resistance gene $\mathrm{mcr}$ - 1 in different genetic backgrounds $[31,32]$. However, in 9953L55, the ISApl1 did not flank any known antibiotic resistance genes.

Nevertheless, both "A. porcitonsillarum" 9953L55 and A. minor $\mathrm{NM} 305^{\mathrm{T}}$ strains possessed a tetracycline resistance operon containing tet(B), which was located on a $\operatorname{Tn} 10$ 


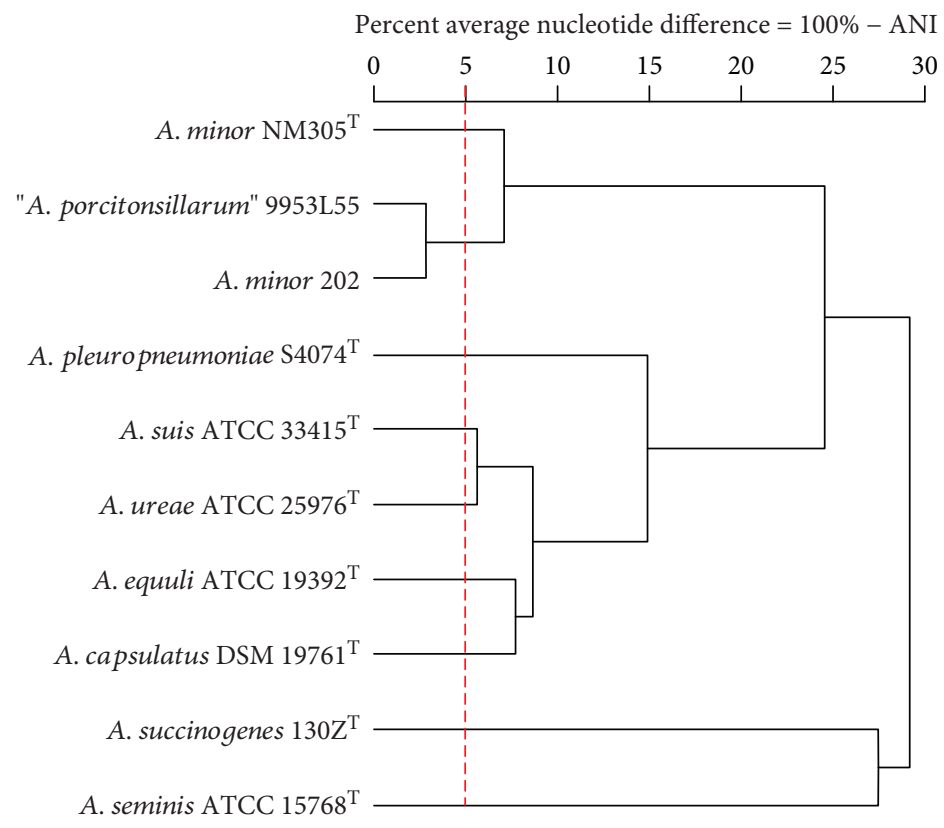

Figure 4: Cluster analysis of average nucleotide identity (ANI) values obtained by BLAST-based pairwise comparisons of 10 Actinobacillus spp. genome sequences. The distance matrix representing the ANI divergence (defined as 100\% - ANI) was used for the complete linkage hierarchical clustering. The vertical dashed line represents the $95 \%$ species cutoff value.

mobile element flanked by two ISVsa5 in opposite orientation [33]. This mobile element, which is widely disseminated among different bacterial species, was also found on integrative conjugative elements in A. pleuropneumoniae (ICEApl1) and other Pasteurellaceae, such as Haemophilus parainfluenzae (ICEHpaT3T1) [34, 35].

Regarding antimicrobial resistance, we note that in " $A$. porcitonsillarum" 9953L55, tet(B) was the only resistance gene identified by the in silico analysis with ResFinder. However, we additionally identified in this strain, as well as in $A$. minor 202, an ortholog encoding a major facilitator superfamily (MFS) transporter ( $\mathrm{LmrB}$ ) potentially associated with lincomycin resistance.

Pairwise comparisons of the genome sequences of these four strains and the type strains of six other Actinobacillus spp. based on ANI confirmed the close relationship between "A. porcitonsillarum" and A. minor (Figure 4). However, ANIb values for " $A$. porcitonsillarum" 9953L55 and A. minor 202 were above the $95 \%$ species criteria between each other, but were below for both strains when compared with $A$. minor NM305 ${ }^{\mathrm{T}}$ (Suppl. Table S1) $[24,36]$, indicating that 9953L55 and 202 may belong to a distinct new species closely related to $A$. minor, as suggested previously $[1,7]$. Since ANIm may be more robust for genomes sharing $>90 \%$ sequence similarity [37], we also implemented ANIm for pairwise comparisons of "A. porcitonsillarum" 9953L55 and both $A$. minor strains. The ANIm values for 9953L55 and 202 correlated well with the ANIb values; that is, both were 97.3\% between each other but $93.6 \%$ when compared with $\mathrm{NM} 305^{\mathrm{T}}$, supporting once more the novel species hypothesis.

The same conclusions were drawn also from is $\mathrm{DDH}$ based on genome BLAST distance phylogeny, with only " $A$. porcitonsillarum" 9953L55 and A. minor 202 exhibiting is $\mathrm{DDH}$ values $>70 \%$, i.e., above the same-species threshold
(Suppl. Table S2) [25]. Intriguingly, in a previous study, DNA-DNA relatedness assessed by a classic DNA-DNA hybridization (DDH) method showed borderline specieslevel values for 202 compared with other A. minor strains and, in particular, DDH values $<70 \%$ and a melting temperature difference $>5^{\circ} \mathrm{C}$ for 202 compared with $\mathrm{NM} 305^{\mathrm{T}}$, already indicating that these two strains may not belong to the same species $[5,38]$. Of note, it has been suggested previously that ANI values $\geq 96 \%$ and is DDH values $>70 \%$ (at the upper $95 \%$ confidence interval) are good predictors of the same-species genomes in Aeromonas spp. [39].

\section{Conclusions}

In conclusion, we implement herein the ONT and Illumina sequencing technologies to obtain the first, complete, and highly accurate genome sequence of an " $A$. porcitonsillarum" strain and highlight its main features and differences compared to those of A. pleuropneumoniae and A. minor. Pairwise genome comparisons of 9953L55 with A. minor 202 and $\mathrm{NM}^{2} 5^{\mathrm{T}}$ based on both ANI and isDDH support previous observations that "A. porcitonsillarum" should be recognized as a new species closely related to $A$. minor, to which strain 202 also belongs. This would be essential to clearly differentiate this nonpathogenic species from the pathogenic A. pleuropneumoniae in diagnostic settings and, consequently, in eradication programs.

\section{Data Availability}

The data used to support the findings of this study are available from the corresponding author upon request. 


\section{Conflicts of Interest}

The authors declare that there is no conflict of interest regarding the publication of this paper.

\section{Acknowledgments}

This work was supported by CTI grant number 25291.2 PFLS-LS. We thank Dr. Marcelo Gottschalk for kindly donating the "A. porcitonsillarum" strain 9953L55 and Dr. Joachim Frey for advice.

\section{Supplementary Materials}

Table S1: the similarity matrix of the Actinobacillus spp. genome sequences based on average nucleotide identity (ANI) of pairwise sequence alignments performed with BLAST (ANIb). Table S2: the isDDH values estimated for "A. porcitonsillarum", A. minor 202, A. minor $\mathrm{NM} 305^{\mathrm{T}}$, and A. pleuropneumoniae $\mathrm{S} 4074^{\mathrm{T}}$. (Supplementary Materials)

\section{References}

[1] M. Gottschalk, A. Broes, K. R. Mittal et al., "Non-pathogenic Actinobacillus isolates antigenically and biochemically similar to Actinobacillus pleuropneumoniae: a novel species?," Veterinary Microbiology, vol. 92, no. 1-2, pp. 87-101, 2003.

[2] E. L. Sassu, J. T. Bossé, T. J. Tobias, M. Gottschalk, P. R. Langford, and I. Hennig-Pauka, "Update on Actinobacillus pleuropneumoniae-knowledge, gaps and challenges," Transboundary and Emerging Diseases, vol. 65, no. S1, pp. 72-90, 2018.

[3] M. Gottschalk, E. Altman, N. Charland, F. De Lasalle, and J. D. Dubreuil, "Evaluation of a saline boiled extract, capsular polysaccharides and long-chain lipopolysaccharides of Actinobacillus pleuropneumoniae serotype 1 as antigens for the serodiagnosis of swine pleuropneumonia," Veterinary Microbiology, vol. 42, no. 2-3, pp. 91-104, 1994.

[4] A. Schaller, S. P. Djordjevic, G. J. Eamens et al., "Identification and detection of Actinobacillus pleuropneumoniae by PCR based on the gene apxIVA," Veterinary Microbiology, vol. 79, no. 1, pp. 47-62, 2001.

[5] K. Møller, V. Fussing, P. A. D. Grimont, B. J. Paster, F. E. Dewhirst, and M. Kilian, "Actinobacillus minor sp. nov., Actinobacillus porcinus sp. nov., and Actinobacillus indolicus sp. nov., three new $\mathrm{V}$ factor-dependent species from the respiratory tract of pigs," International Journal of Systematic Bacteriology, vol. 46, no. 4, pp. 951-956, 1996.

[6] G. Arya and D. F. Niven, "Production of haemolysins by strains of the Actinobacillus minor/"porcitonsillarum" complex," Veterinary Microbiology, vol. 141, no. 3-4, pp. 332$341,2010$.

[7] P. Kuhnert and H. Christensen, "International Committee on Systematics of Prokaryotes. Subcommittee on the taxonomy of Pasteurellaceae: minutes of the meetings, 6 August 2008, Istanbul, Turkey," International Journal of Systematic and Evolutionary Microbiology, vol. 59, no. 1, pp. 202-203, 2009.

[8] P. Kielstein, H. Wuthe, O. Angen, R. Mutters, and P. Ahrens, "Phenotypic and genetic characterization of NAD-dependent Pasteurellaceae from the respiratory tract of pigs and their possible pathogenetic importance," Veterinary Microbiology, vol. 81, no. 3, pp. 243-255, 2001.

[9] J. Sambrook and D. W. Russell, "Purification of nucleic acids by extraction with phenol:chloroform," Cold Spring Harbor Protocols, vol. 2006, no. 1, 2006.

[10] S. Koren, B. P. Walenz, K. Berlin, J. R. Miller, and A. M. Phillippy, "Canu: scalable and accurate long-read assembly via adaptive $k$-mer weighting and repeat separation," Genome Research, vol. 27, no. 5, pp. 722-736, 2017.

[11] B. J. Walker, T. Abeel, T. Shea et al., "Pilon: an integrated tool for comprehensive microbial variant detection and genome assembly improvement," PLoS One, vol. 9, no. 11, article e112963, 2014.

[12] T. Seemann, "Prokka: rapid prokaryotic genome annotation," Bioinformatics, vol. 30, no. 14, pp. 2068-2069, 2014.

[13] D. Antipov, N. Hartwick, M. Shen, M. Raiko, A. Lapidus, and P. A. Pevzner, "plasmidSPAdes: assembling plasmids from whole genome sequencing data," Bioinformatics, vol. 32, no. 22, pp. 3380-3387, 2016.

[14] A. E. Darling, B. Mau, and N. T. Perna, "progressiveMauve: multiple genome alignment with gene gain, loss and rearrangement," PLoS One, vol. 5, no. 6, article e11147, 2010.

[15] Y. Wang, D. Coleman-Derr, G. Chen, and Y. Q. Gu, "OrthoVenn: a web server for genome wide comparison and annotation of orthologous clusters across multiple species," Nucleic Acids Research, vol. 43, no. W1, pp. W78-W84, 2015.

[16] E. Zankari, H. Hasman, S. Cosentino et al., "Identification of acquired antimicrobial resistance genes," The Journal of Antimicrobial Chemotherapy, vol. 67, no. 11, pp. 2640-2644, 2012.

[17] A. Carattoli, E. Zankari, A. García-Fernández et al., "In silico detection and typing of plasmids using PlasmidFinder and plasmid multilocus sequence typing," Antimicrobial Agents and Chemotherapy, vol. 58, no. 7, pp. 3895-3903, 2014.

[18] P. Siguier, J. Perochon, L. Lestrade, J. Mahillon, and M. Chandler, "ISfinder: the reference centre for bacterial insertion sequences," Nucleic Acids Research, vol. 34, no. 90001, pp. D32-D36, 2006.

[19] Q. Zhang and Y. Ye, "Not all predicted CRISPR-Cas systems are equal: isolated cas genes and classes of CRISPR like elements," BMC Bioinformatics, vol. 18, no. 1, p. 92, 2017.

[20] D. Arndt, J. R. Grant, A. Marcu et al., "PHASTER: a better, faster version of the PHAST phage search tool," Nucleic Acids Research, vol. 44, no. W1, pp. W16-W21, 2016.

[21] N. F. Alikhan, N. K. Petty, N. L. Ben Zakour, and S. A. Beatson, "BLAST Ring Image Generator (BRIG): simple prokaryote genome comparisons," BMC Genomics, vol. 12, no. 1, p. 402, 2011.

[22] J. Goris, J. A. Klappenbach, P. Vandamme, T. Coenye, K. T. Konstantinidis, and J. M. Tiedje, "DNA-DNA hybridization values and their relationship to whole-genome sequence similarities," International Journal of Systematic and Evolutionary Microbiology, vol. 57, no. 1, pp. 81-91, 2007.

[23] M. Richter, R. Rosselló-Móra, F. Oliver Glöckner, and J. Peplies, "JSpeciesWS: a web server for prokaryotic species circumscription based on pairwise genome comparison," Bioinformatics, vol. 32, no. 6, pp. 929-931, 2016. 
[24] C. O'Flynn, O. Deusch, A. E. Darling et al., "Comparative genomics of the genus Porphyromonas identifies adaptations for heme synthesis within the prevalent canine oral species Porphyromonas cangingivalis," Genome Biology and Evolution, vol. 7, no. 12, pp. 3397-3413, 2015.

[25] J. P. Meier-Kolthoff, A. F. Auch, H.-P. Klenk, and M. Göker, "Genome sequence-based species delimitation with confidence intervals and improved distance functions," BMC Bioinformatics, vol. 14, no. 1, p. 60, 2013.

[26] A. F. Auch, M. von Jan, H. P. Klenk, and M. Göker, "Digital DNA-DNA hybridization for microbial species delineation by means of genome-to-genome sequence comparison," Standards in Genomic Sciences, vol. 2, no. 1, pp. 117-134, 2010.

[27] V. J. Rapp, R. F. Ross, and T. F. Young, "Characterization of Haemophilus spp. isolated from healthy swine and evaluation of cross-reactivity of complement-fixing antibodies to Haemophilus pleuropneumoniae and Haemophilus taxon "minor group"," Journal of Clinical Microbiology, vol. 22, no. 6, pp. 945-950, 1985.

[28] R. Jansen, J. Briaire, E. M. Kamp, A. L. Gielkens, and M. A. Smits, "The CAMP effect of Actinobacillus pleuropneumoniae is caused by Apx toxins," FEMS Microbiology Letters, vol. 126, no. 2, pp. 139-143, 1995.

[29] J. Frey, "Virulence in Actinobacillus pleuropneumoniae and RTX toxins," Trends in Microbiology, vol. 3, no. 7, pp. 257261, 1995.

[30] P. Kuhnert, Y. Schlatter, and J. Frey, "Characterization of the type I secretion system of the RTX toxin ApxII in "Actinobacillus porcitonsillarum"," Veterinary Microbiology, vol. 107, no. 3-4, pp. 225-232, 2005.

[31] L. Poirel, N. Kieffer, and P. Nordmann, "In vitro study of ISApl1-mediated mobilization of the colistin resistance gene mor-1," Antimicrobial Agents and Chemotherapy, vol. 61, no. 7, 2017.

[32] V. Donà, O. J. Bernasconi, J. Pires et al., "Heterogeneous genetic location of $m c r-1$ in colistin-resistant Escherichia coli isolates from humans and retail chicken meat in Switzerland: emergence of mcr-1-carrying IncK2 plasmids," Antimicrobial Agents and Chemotherapy, vol. 61, no. 11, 2017.

[33] T. D. Lawley, V. Burland, and D. E. Taylor, "Analysis of the complete nucleotide sequence of the tetracycline-resistance transposon Tn10," Plasmid, vol. 43, no. 3, pp. 235-239, 2000.

[34] J. T. Bossé, Y. Li, R. F. Crespo et al., "ICEApl1, an integrative conjugative element related to ICEHin1056, identified in the pig pathogen Actinobacillus pleuropneumoniae," Frontiers in Microbiology, vol. 7, p. 810, 2016.

[35] M. Juhas, P. M. Power, R. M. Harding et al., "Sequence and functional analyses of Haemophilus spp. genomic islands," Genome Biology, vol. 8, no. 11, article R237, 2007.

[36] J. Z. Chan, M. R. Halachev, N. J. Loman, C. Constantinidou, and M. J. Pallen, "Defining bacterial species in the genomic era: insights from the genus Acinetobacter," BMC Microbiology, vol. 12, no. 1, p. 302, 2012.

[37] M. Richter and R. Rosselló-Móra, "Shifting the genomic gold standard for the prokaryotic species definition," Proceedings of the National Academy of Sciences of the United States of America, vol. 106, no. 45, pp. 19126-19131, 2009.
[38] L. G. Wayne, "International Committee on Systematic Bacteriology: announcement of the report of the ad hoc Committee on Reconciliation of Approaches to Bacterial Systematics," Zentralblatt für Bakteriologie, Mikrobiologie und Hygiene. Series A: Medical Microbiology, Infectious Diseases, Virology, Parasitology, vol. 268, no. 4, pp. 433-434, 1988.

[39] S. M. Colston, M. S. Fullmer, L. Beka, B. Lamy, J. P. Gogarten, and J. Graf, "Bioinformatic genome comparisons for taxonomic and phylogenetic assignments using Aeromonas as a test case," MBio, vol. 5, no. 6, article e02136, 2014. 


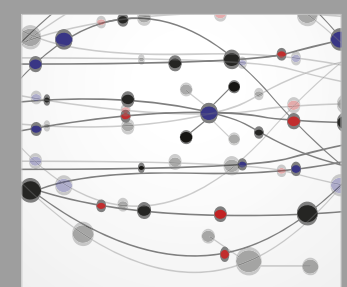

The Scientific World Journal
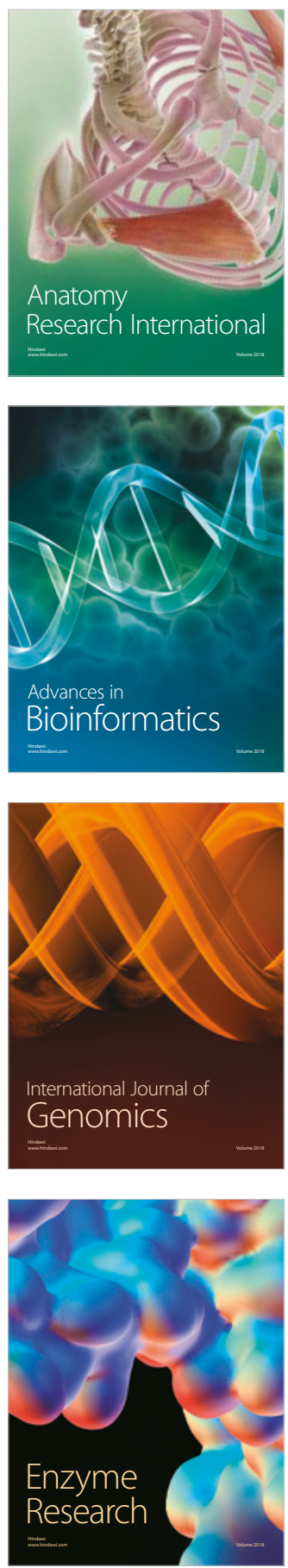
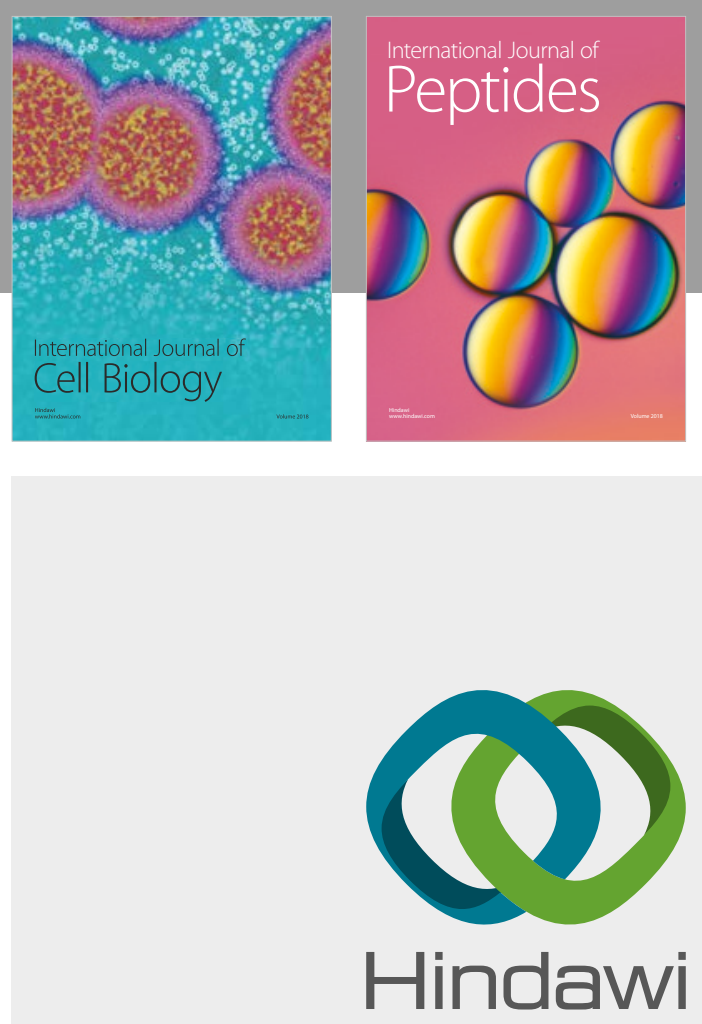

Submit your manuscripts at

www.hindawi.com
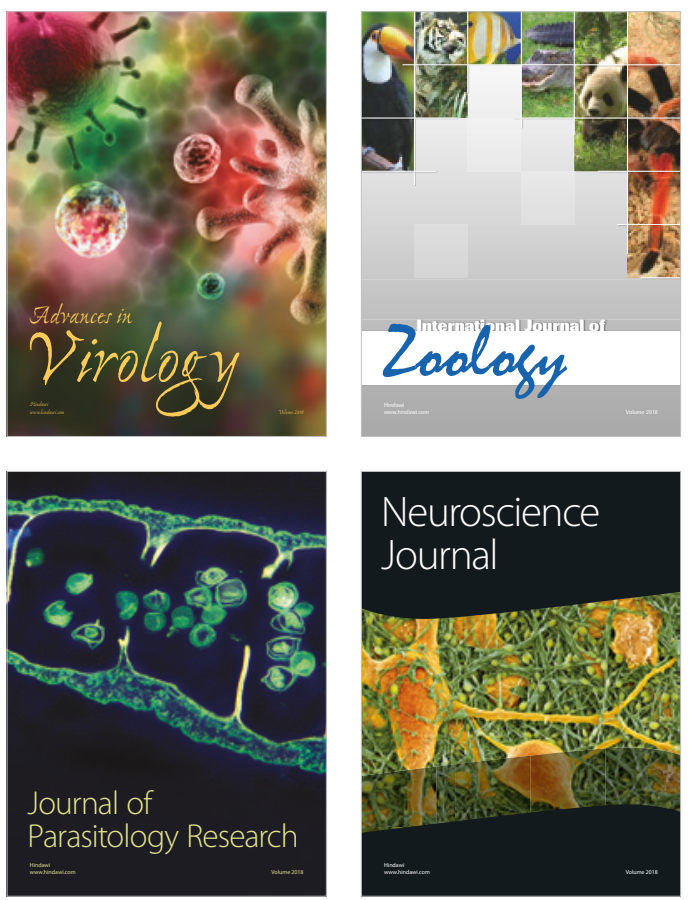
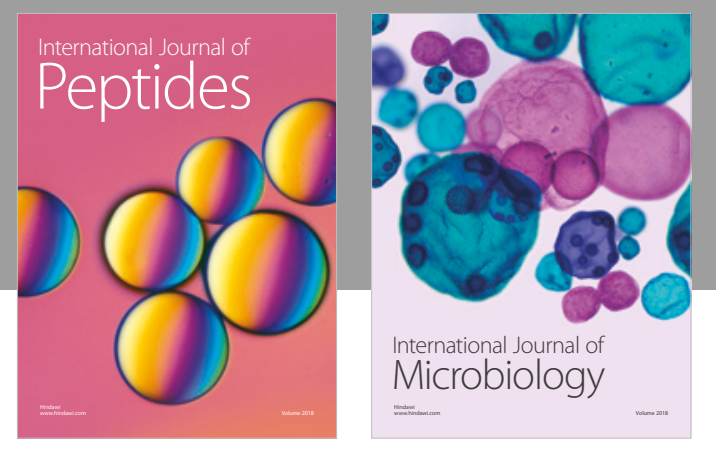

nternational Journal of Microbiology
Journal of
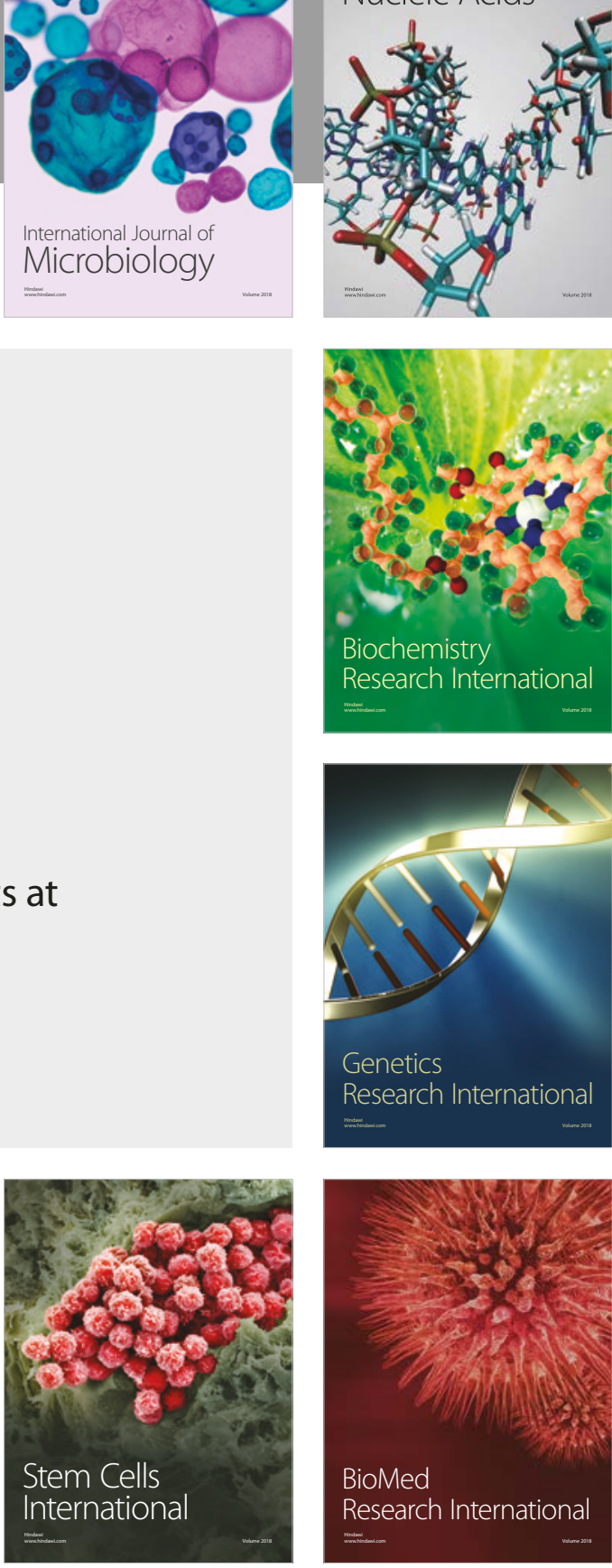
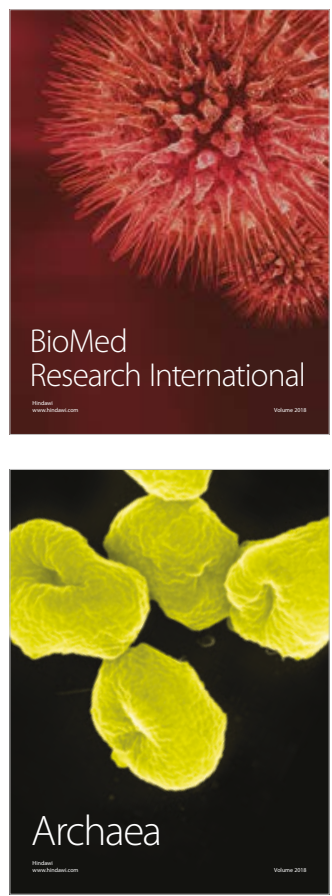